ARTICLE

httpst//doi.org/10.1038/s41467-020-15682-2

\title{
Broadband frequency translation through time refraction in an epsilon-near-zero material
}

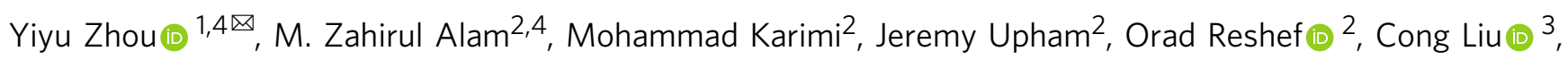
Alan E. Willner ${ }^{3} \&$ Robert W. Boyd (1) ${ }^{1,2}$

Space-time duality in paraxial optical wave propagation implies the existence of intriguing effects when light interacts with a material exhibiting two refractive indexes separated by a boundary in time. The direct consequence of such time-refraction effect is a change in the frequency of light while leaving the wavevector unchanged. Here, we experimentally show that the effect of time refraction is significantly enhanced in an epsilon-near-zero (ENZ) medium as a consequence of the optically induced unity-order refractive index change in a sub-picosecond time scale. Specifically, we demonstrate broadband and controllable shift (up to $14.9 \mathrm{THz}$ ) in the frequency of a light beam using a time-varying subwavelength-thick indium tin oxide (ITO) film in its ENZ spectral range. Our findings hint at the possibility of designing $(3+1)$ D metamaterials by incorporating time-varying bulk ENZ materials, and they present a unique playground to investigate various novel effects in the time domain.

\footnotetext{
${ }^{1}$ The Institute of Optics, University of Rochester, Rochester, NY 14627, USA. ${ }^{2}$ Department of Physics, University of Ottawa, Ottawa, ON K1N 6N5, Canada. ${ }^{3}$ Department of Electrical Engineering, University of Southern California, Los Angeles, CA 90089, USA. ${ }^{4}$ These authors contributed equally: Yiyu Zhou,

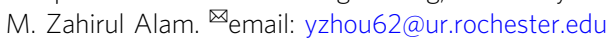


$\mathrm{M}$ axwell's equations describe how an electromagnetic wave is modified by a material. The spatial boundary condition associated with Maxwell's equations can be used to derive the well-known Fresnel equations and Snell's law. A spatial variation in refractive index leads to reflection and refraction of a light beam incident on the boundary. As a consequence, the wavevector of the transmitted light changes, whereas the frequency is conserved. The spatial boundary can be abrupt (nonadiabatic) in refractive index variation such as at a glass-air interface. Or, the boundary can be smoothly varying, i.e., adiabatic in space, such as in a gradient-index lens. In both cases, the refracted beam of light must have a different $k$-vector (Fig. 1a), where $|k|=2 \pi n / \lambda, n$ is the refractive index of the medium, and $\lambda$ is vacuum wavelength of light. As the equations describing the paraxial wave propagation are unchanged upon the interchange of time and a spatial coordinate, one can define a boundary of refractive index in the time coordinate in a dual fashion to that in the spatial coordinates ${ }^{1-5}$. This effect is known as time refraction.

The concept of time refraction is presented in Fig. 1a. Let us assume that an optical pulse of frequency $f_{1}$ is traveling in a dispersionless medium with a refractive index of $n_{1}$. At $t=t_{1}$ the refractive index changes from $n_{1}$ to $n_{2}$. As a consequence of the broken time translation symmetry, the frequency of light has to change because of the change in the refractive index while leaving

a
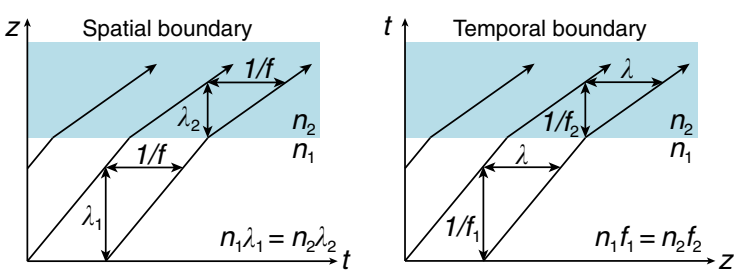

b
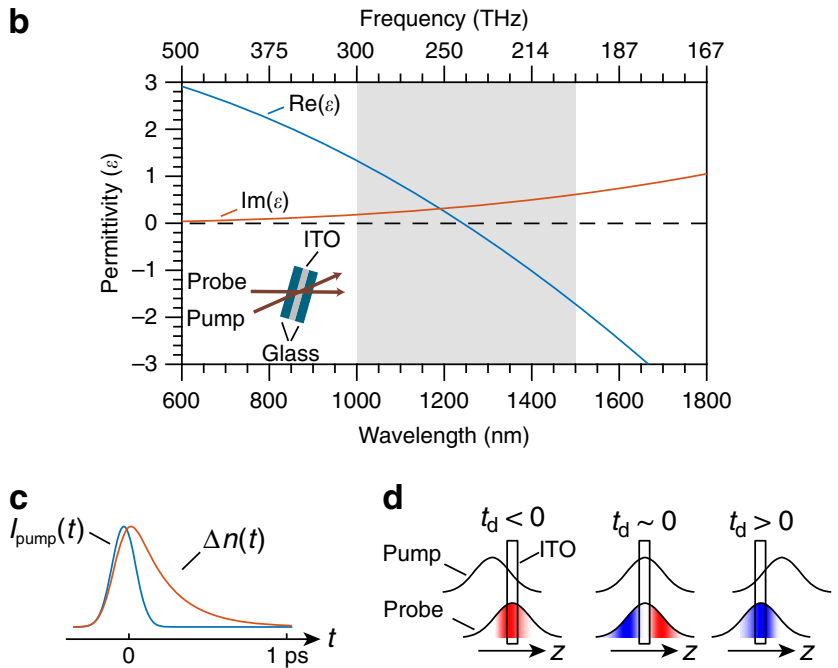

Fig. 1 Concept of time refraction. a A spatial boundary defined by a refractive index change from $n_{1}$ to $n_{2}$ leads to a change in the wavevector of a light beam as it passes through the boundary and is described by $n_{1} \lambda_{1}=$ $n_{2} \lambda_{2}$ (left panel). A refractive index boundary defined in time leads to timerefraction effect of a light beam as it passes through the boundary and is described by $n_{1} f_{1}=n_{2} f_{2}$ (right panel). Here $f$ is the frequency of light waves in the medium. b The permittivity of an ITO film used in the experiment. The inset shows the simplified experimental setup and the shaded region shows the spectral range of interest in this work. c Simplified illustration of the temporal index change $\Delta n(t)$ of ITO excited by a pump pulse. d The frequency of the probe redshifts (blueshifts) if the pump beam lags (leads) the probe. At near-zero delay both redshift and blueshift can occur. the wavevector unchanged ${ }^{6}$. The change in frequency, according to the dispersion relation $c / f=n \lambda^{7}$, can be expressed as $n_{1} f_{1}=n_{2} f_{2}$ $=\left(n_{1}+\Delta n\right)\left(f_{1}+\Delta f\right)$, where $\Delta f=f_{2}-f_{1}$ is the change in the frequency of light after it encounters the temporal boundary; $\Delta n=$ $n_{2}-n_{1}$ is the change in the refractive index; and $c$ is the speed of light in vacuum. Consequently, we can express the change in frequency as $\Delta f=-\Delta n \cdot f_{1} /\left(n_{1}+\Delta n\right)$. Thus, the frequency shift may be red (blue) if the change in index $\Delta n$ is positive (negative). This effect is strongest when $\Delta n /\left(n_{1}+\Delta n\right)$ is large. In a regular dielectric medium such as silicon ${ }^{8}, \Delta n /\left(n_{1}+\Delta n\right)$ can only be on the order of $10^{-3}$. In contrast, in a highly nonlinear low-index medium, $\Delta n /\left(n_{1}+\Delta n\right)$ can approach unity due to the near-zero linear refractive index $n_{1}$ and the large nonlinear index change $\Delta n^{9-11}$. Thus, a highly nonlinear low-index medium is a natural platform with which to generate a large frequency translation using time refraction. In addition to frequency conversion, a timevarying medium with a large index change can also be used to investigate many novel effects in the time domain such as alloptical nonreciprocity $^{12,13}$, negative refraction ${ }^{14}$, photonic topological insulators ${ }^{15}$, photonic time crystals ${ }^{16}$, achromatic optical switches ${ }^{17}$, and the dynamic Casimir effect ${ }^{18}$.

A number of effects have been used to experimentally implement a time-varying medium, such as free-carrier dispersion ${ }^{19-24}$, Kerr nonlinearity ${ }^{25-28}$, laser-induced plasma ${ }^{29-32}$, and optomechanical interaction ${ }^{33}$. The magnitude of frequency conversion in a time-varying medium fundamentally depends on the available index change. This is in contrast to other nonlinear optical effects such as four-wave mixing ${ }^{8}$, where the constraints of weak nonlinearity can be almost entirely overcome through use of a very long interaction length ${ }^{34}$. Resonant structures such as microring resonators and slow-light photonic crystals, tend to exhibit enhanced sensitivity to the change in the material's refractive index. Such resonant structures can be used to somewhat sidestep the restrictions imposed by the intrinsically low nonlinearity of materials to obtain appreciable adiabatic frequency conversion (AFC) ${ }^{19-22,35-39}$. Using these techniques, adiabatic frequency conversions up to $280 \mathrm{GHz}$ (or $\sim 0.145 \%$ of the carrier frequency) have been previously demonstrated ${ }^{20}$. Nevertheless, all prior demonstrations of AFC have exhibited the following limitations: narrow operational bandwidth $20,34,38,39$; relatively long interaction length $19,21-25,33,36,37,40$; limited tunability with respect to the magnitude and sign of shift ${ }^{19-24,40}$; the requirement of inhomogeneous structure limiting wide adoptions into various platforms ${ }^{19-24,33,35-39}$; and the possible requirement of out-of-plane above-bandgap excitation pulses ${ }^{20}$.

Here, we show that we can simultaneously overcome all of the above-mentioned shortcomings by using a homogeneous and isotropic epsilon-near-zero (ENZ) medium of subwavelength thickness. Using a series of pump-probe measurements, we demonstrate optically controlled total frequency translations of a near-infrared beam of up to $14.9 \mathrm{THz}$ (redshift of $11.1 \mathrm{THz}$ and blueshift of $3.8 \mathrm{THz}$ ) - that is, over $6 \%$ of the bandwidth of the carrier frequency-using a 620-nm-thick ITO film. The effect of frequency translation is broadband in nature, i.e., the central wavelength of the degenerate input pump and probe pulses can be tuned over a $500 \mathrm{~nm}$ range. We also find that the effect is maximum near the zero-permittivity wavelength of ITO.

\section{Results}

Nonlinear optical response of the ENZ material. An ENZ material is defined as a medium that has a near-zero linear permittivity, and consequently low linear refractive index. The near-zero permittivity in such a medium leads to highly nonintuitive linear effects ${ }^{41-43}$ and strong nonlinear light-matter interactions ${ }^{9,44-47}$. In order to implement a temporal boundary 
with a large index change, we make use of the large and ultrafast optically induced change in refractive index of a $620 \mathrm{~nm}$ thick ITO film in its near-zero-permittivity spectral range. ITO is a degenerately doped semiconductor and near its zero-permittivity wavelength $(1240 \mathrm{~nm})$, the linear permittivity of the ITO sample can be well described by the Drude model (Fig. 1b). The temporal nonlinear optical response of ITO can be described by the twotemperature model when excited by an optical pulse with a central wavelength close to the ENZ region ${ }^{9,44}$. The optical excitation of ITO near the ENZ region leads to a strong modification of the Fermi-Dirac distribution of the conduction band electrons. The highly nonequilibrium distribution of electrons, within the formalism of the Drude model, leads to an effective redshift of the plasma frequency owing to the momentumdependent effective mass of the electrons. According to the twotemperature model, the rise time of the change in the refractive index is limited by the thermalization time of the conduction band electrons owing to electron-electron scattering. The rise time also depends on the energy deposition rate in the ITO film and thus has a strong dependence on the temporal envelope of the pump pulse. Once the pump pulse peak leaves the ITO film, the index returns to the initial value within a sub-picosecond time scale through electron-phonon coupling (Fig. 1c). Owing to the time-dependent nature of the index change induced by the intensity of the pump pulse, the frequency of probe pulse can be redshifted or blueshifted depending on the pump-probe delay time (see Fig. 1d).

Measurements at the near-zero-permittivity wavelength. In order to measure the magnitude of the frequency translation using ITO, we performed a set of degenerate pump-probe experiments with $\sim 120$ fs pulses and recorded the spectra of the probe beam as a function of the delay between the pump and the probe for varying pump intensities. The ITO film has two 1.1$\mathrm{mm}$-thick glass slabs on both sides. Both pump and probe beams are $p$-polarized, and the intensity of the probe beam is kept low to avoid nonlinear effects (See Methods and Supplementary Note 1 for more details). The results for $\lambda_{0}=\lambda_{\text {pump }}=\lambda_{\text {probe }}=1235 \mathrm{~nm}$ at the pump-probe delay time of $\pm 60 \mathrm{fs}$ is shown in Fig. 2. The pump induces a nonlinear change in the refractive index of ITO with a rate that depends on the pump intensity, the temporal

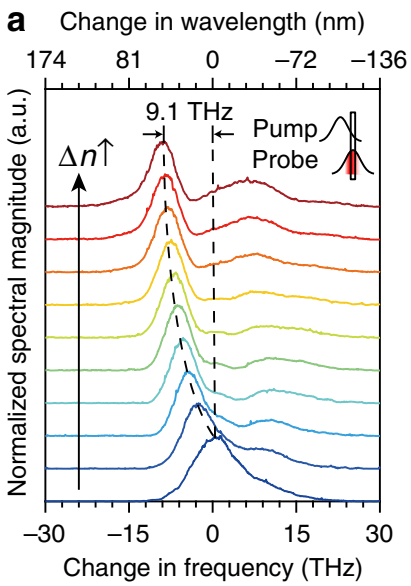

\section{b Change in wavelength $(\mathrm{nm})$}
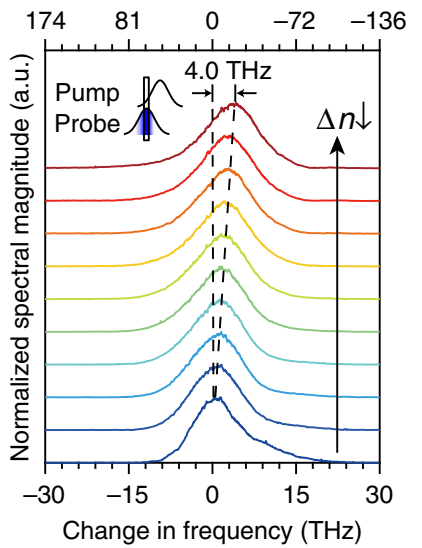

Fig. 2 Pump-induced frequency translation at a fixed delay time. $a-b$ The frequency of a $1235 \mathrm{~nm}$ probe beam redshifts at the delay time $t_{\mathrm{d}}=-60 \mathrm{fs}$ in $\mathbf{a}$ and blueshifts at the delay time $t_{\mathrm{d}}=60 \mathrm{fs}$ in $\mathbf{b}$. The insets show the relative position of the pump and the probe. The top-most (bottom-most) spectra in both panels correspond to the largest (zero) pump intensity and, consequently, the largest (zero) change in the refractive index. envelope of the pump, and the intrinsic nonlinear dynamics of the ITO. When the pump pulse is delayed with respect to the probe, i.e., pump-probe delay time $t_{\mathrm{d}}<0$, the probe experiences a rising refractive index and thus its spectrum redshifts (Fig. 2a). If the probe reaches the ITO after the peak of the pump pulse is passed $\left(t_{\mathrm{d}}>0\right)$, it experiences a falling refractive index change and the spectrum of the probe blueshifts (Fig. $2 \mathrm{~b}$ ). We also note that for $t_{\mathrm{d}} \approx 0$ both blueshift and redshift can occur (Fig. 1d). As the thickness of the ITO film is only $620 \mathrm{~nm}, 120 \mathrm{fs}$ pump, and the probe pulses never reside entirely within the ITO thin film (Fig. 1d). Thus, the magnitude of the frequency shift of the probe pulse becomes dependent on the index change rate $\Delta n / \Delta t$ it experiences while transiting through the ITO film. We extract the effective values of the index change rate based on the experimental data through numerical simulations (see Supplementary Note 2). In numerical simulation we use the slowly varying envelope approximation and, as a result, the predictions of our model are only dependent on the envelope-averaged dynamics of the ITO.

We find that both the pump intensity and the value of the pump-probe delay time modify the spectra of the transmitted probe. We present the results for $\lambda_{0}=1235 \mathrm{~nm}$ for three pump intensities in Fig. 3a-c. In general, the time refraction leads to the modification of amplitude, bandwidth, temporal width, and the carrier frequency of the probe pulse (see Supplementary Note 3). In order to focus on the spectral shift, the magnitude of spectrum for each pump-probe delay value is individually normalized in Fig. 3. We find that when the absolute value of the pump-probe delay time $\left|t_{\mathrm{d}}\right|$ is increased, the magnitude of the frequency translation for the probe decreases. Furthermore, when pumpprobe delays are small, the leading portion of the probe pulse experiences an increase in refractive index (thus redshifts), whereas the trailing portion experiences a decrease of refractive index (thus blueshifts). This is evident in Fig. $3 \mathrm{a}-\mathrm{c}$ by the presence of two peaks at $t_{\mathrm{d}} \approx 0$. For a fixed pump-probe delay time an increase in pump intensity leads to larger change in index and, as a result, a larger shift in the central frequency of the probe pulse. Furthermore, we find that the fall time of the index change is slower than the rise time of the index change. The fall time of the index change is longer because it-within the formalism of the two-temperature model-is dictated by the intrinsic electron-phonon coupling rate, the maximum temperature of the conduction band electrons, and the thermodynamical properties of the lattice. As a result, the rate of decrease in index after the pump leaves the ITO film is smaller compared with that of the rising edge, and therefore the magnitude of the achievable redshift for a constant pump intensity is larger than the achievable blueshift. At a sufficiently high pump intensity, we observe an appearance of a large blueshifted spectral peak when the pump is at $1235 \mathrm{~nm}$ owing to higher-order nonlinear optical effects. At a peak pump intensity of $483 \mathrm{GW} \mathrm{cm}^{-2}$ the blueshift can be as large as $10.6 \mathrm{THz}(\sim 52 \mathrm{~nm}$ in wavelength), and the total maximum frequency translation can be larger than $20 \mathrm{THz}$ (see Supplementary Note 4). This value corresponds to a fractional frequency shift $\left(\Delta f / f_{0}\right)$ of $\sim 9 \%$.

We model the time-refraction effect in ITO using the nonlinear Schrödinger equation, and the split-step Fourier method is used to numerically solve the Schrödinger equation ${ }^{48}$. We use an iterative algorithm to calculate the approximate shape of the time-varying nonlinear phase variations induced by the index change to fit the experimentally measured spectra (see Supplementary Note 2). The simulation results are shown in Fig. 3d-f. Our numerical model is in excellent agreement with the experimental data, confirming that the origin of the shift is owing to the rapid change of index experienced by the probe pulse while transiting through the ITO sample. 
a

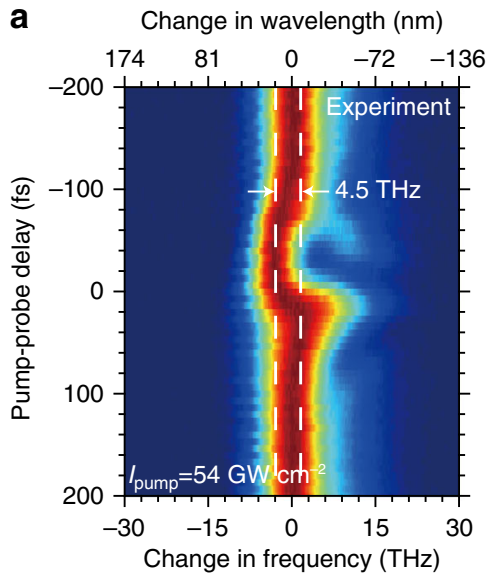

d

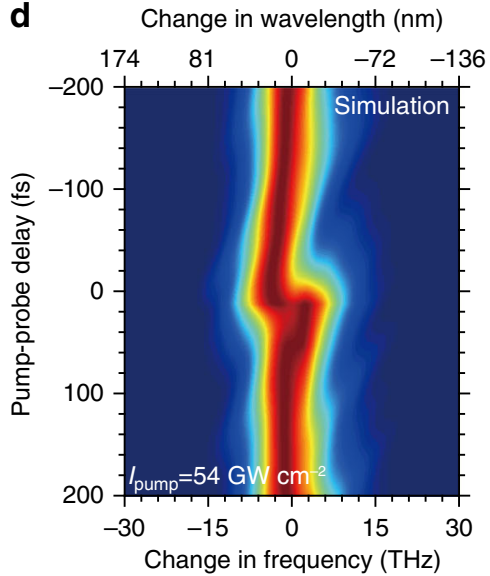

b Change in wavelength $(\mathrm{nm})$

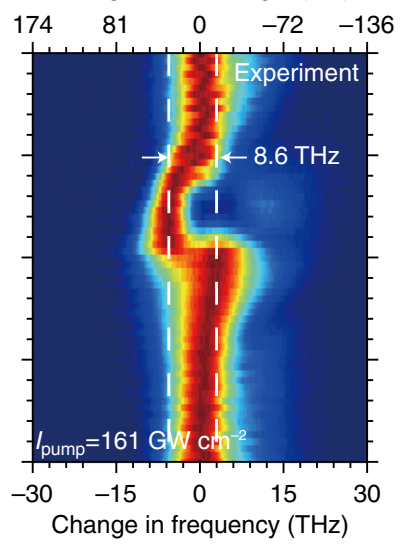

e Change in wavelength $(\mathrm{nm})$
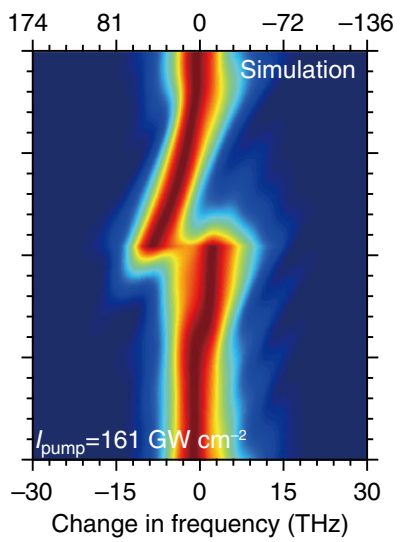

C Change in wavelength $(\mathrm{nm})$
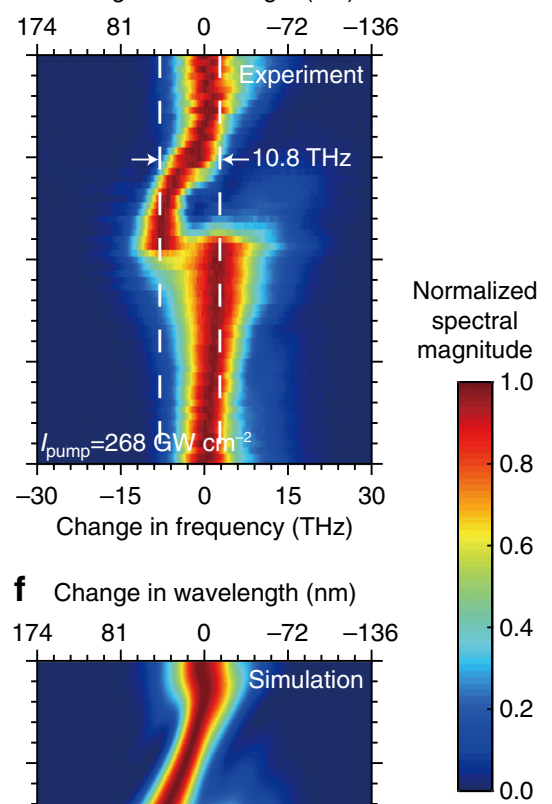

f Change in wavelength $(\mathrm{nm})$
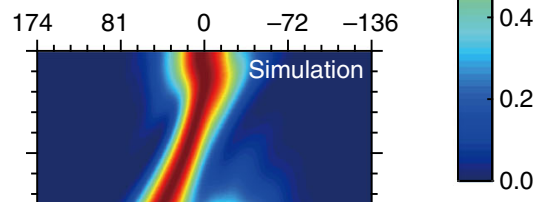

.0

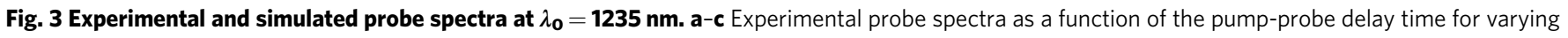
pump intensities. The spectral magnitude for each pump-probe delay is normalized individually. d-f The corresponding numerically simulated probe

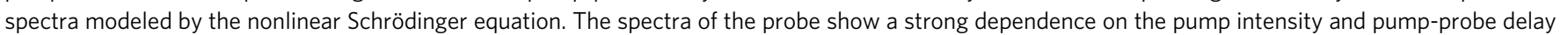
time. For a pump intensity of $268 \mathrm{GW} \mathrm{cm}^{-2}$, the total frequency translation at this wavelength is $10.8 \mathrm{THz}$.

Measurements over a broad spectral range. Next, we investigate the dynamics away from the zero-permittivity wavelengths. We repeat the measurements at different excitation wavelengths from $\lambda_{0}=1000 \mathrm{~nm}-1500 \mathrm{~nm}$. For each excitation wavelength and pump intensity, we extract the maximum frequency translation of the probe over a range of pump-probe delay time (see Supplementary Note 5). We summarize the wavelength- and intensitydependent maximum frequency translations in Fig. 4a-e. Here, we limit the pump intensities to avoid the occurrence of significant higher-order nonlinearities. Our results reveal a number of trends. First, both the total achievable frequency translation (redshift and blueshift) and the maximum achievable redshift for a constant pump intensity are the highest near $1235 \mathrm{~nm}$ (where $\operatorname{Re}(\varepsilon) \approx 0$ ) than at other wavelengths. For example, at $\lambda_{0}=1495 \mathrm{~nm}$ the measured maximum magnitude of the redshift $(5.4 \mathrm{THz})$ is a factor of two smaller than what can be achieved at $\lambda_{0}=1235 \mathrm{~nm}$ using a lower pump intensity. Nevertheless, we find that the total maximum fractional frequency translation $\left(\Delta f / f_{0}\right)$ at near-zero permittivity is unprecedentedly large (Fig. $4 \mathrm{f}$ ). The maximum total frequency translation of $14.9 \mathrm{THz}$ (redshift of $11.1 \mathrm{THz}$ and blueshift of $3.8 \mathrm{THz}$ ) at $\lambda_{0}=1235 \mathrm{~nm}$ (redshift plus blueshift) is over 53 times larger than what was achieved using a silicon ring resonator of a $6 \mu \mathrm{m}$ diameter exhibiting a $Q$-factor greater than $18,000^{20}$. In contrast, the propagation distance in our material is only $620 \mathrm{~nm}$ which is 30 times shorter in physical length and four orders of magnitude smaller than the effective interaction length in a high- $Q$ cavity. Moreover, our results show the operation bandwidth of ITO is much larger than what can be achieved using high- $Q$ resonant structures.

\section{Discussion}

As the refractive index of the ENZ material depends on the intensity of the pump, the work presented here may be formally described by cross-phase modulation with a delayed response ${ }^{8}$. However, the concept of time refraction is independent of the source type of the index change (e.g., thermally, optomechanically, or electrically induced index change) and is a more general effect than the simple cross-phase modulation that arises when the temporal boundary is specifically induced by an optical pulse. Furthermore, in contrast to a typical four-wave-mixingbased frequency conversion, the frequency shift obtained through time refraction does not depend on the frequency difference between the pump and the probe and is completely free from phase-mismatching. Although in this work the pump and the probe are frequency degenerate and produced from the same source using a beam splitter, it is not necessary for the beams to be frequency degenerate. Nevertheless, the maximum frequency shift with minimum energy expenditure can be achieved when both the pump and the probe lie within the ENZ spectral range. As the maximum index change happens at the zero-permittivity wavelength, the probe will undergo maximum frequency shift if its wavelength is at or near the zeropermittivity wavelength, whereas the energy expenditure will be 

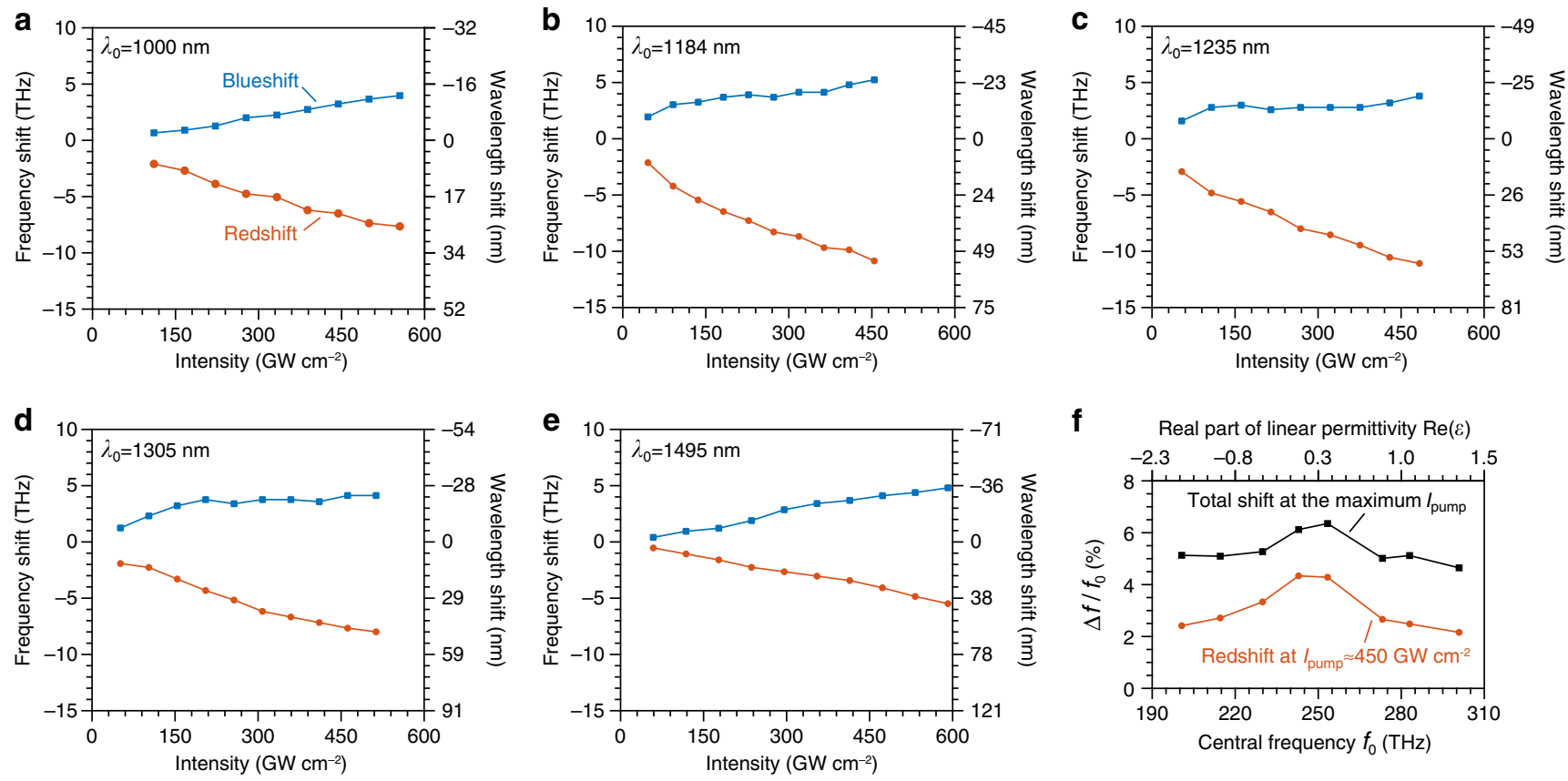

$\mathbf{f}$

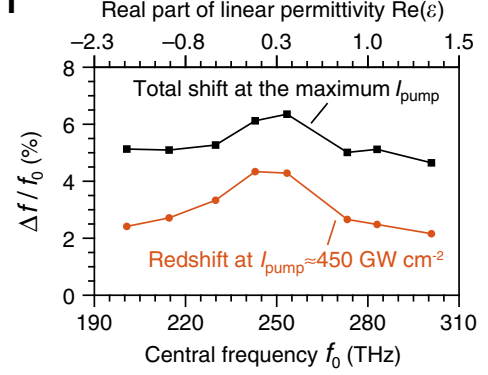

Fig. 4 Wavelength-dependent time-refraction effect. a-e Experimentally measured maximum redshifts and blueshifts at different wavelengths $\lambda_{0}$ as a function of peak pump intensities. $\mathbf{f}$ The red line denotes the fractional redshift $\left|\Delta f_{\text {red }}\right| / f_{0}$ as a function of probe beam's central frequency $f_{0}$ at a peak pump intensity $\sim 450 \mathrm{GW} \mathrm{cm}^{-2}$. The real part of the linear permittivity $\operatorname{Re}(\varepsilon)$ of ITO film at the corresponding central frequency $f_{0}$ is shown in the top axis. The black line shows the total fractional shift (redshift plus blueshift) measured at the maximum pump intensities before the onset of higher-order nonlinear optical effects. We find that both the maximum fractional redshift and the total shift occur near the zero-permittivity wavelength.

minimum if the wavelength of pump is also at or near the zeropermittivity wavelength.

In conclusion, we have shown that a subwavelength-thick ITO film can be used to obtain unprecedentedly large $(\sim 6.5 \%$ of the carrier frequency), broadband and tunable frequency translation. The large time-refraction effect in the ENZ material raises the intriguing possibility of wavelength conversion over an octave using a time-varying ENZ medium. The magnitude of the frequency translation is primarily limited by the linear loss, higherorder nonlinear optical effects, dispersion, and the interplay between the pulse width and the interaction time. We note that the ENZ spectral region of ITO and other conducting oxides can be tuned at any wavelength between $1 \mu \mathrm{m}$ and $3 \mu \mathrm{m}$ by choosing the appropriate doping level ${ }^{49,50}$. Furthermore, because the effect is present in a bulk, homogeneous and isotropic material, one can engineer nanostructures incorporating ENZ media such as plasmonic waveguides, photonic crystal waveguides, and dynamic metasurfaces to arbitrarily control the sign and the magnitude of the frequency shift in order to build efficient octave-spanning frequency tuners while simultaneously lowering the required pump power by a few orders of magnitude ${ }^{9}$. For example, an appropriately engineered ITO-based platform can be used to shift an entire band of optical signals in the frequency domain. Such devices may find practical usage in quantum communication protocols requiring conversion of visible photons to infrared ${ }^{51}$ and in classical coherent optical communications ${ }^{52,53}$. We anticipate that the large time-refraction effect, we report here, can be exploited to engineer magnet-free nonreciprocal devices ${ }^{54,55}$, spatiotemporal metasurfaces ${ }^{13}$, and to investigate photonic time crystals and other topological effects in the time domain ${ }^{16,56}$ using free-space or on-chip ENZ-based structures.

\section{Methods}

Measurements. We use a tunable optical parametric amplifier (OPA) pumped by an amplified Ti:sapphire laser of $\sim 120 \mathrm{fs}$ for the experiments. The output of the OPA is split into two beams to produce the degenerate pump and probe beams using a pellicle beam splitter. Both beams are rendered $p$-polarized. The pump beam is focused onto the sample by a $25 \mathrm{~cm}$ lens yielding to a spot size of $\sim 100 \mu \mathrm{m}$. The probe beam is focused by a $10 \mathrm{~cm}$ lens and its spot diameter is $\sim 45 \mu \mathrm{m}$ at $1235 \mathrm{~nm}$. Although the spot size can change when the wavelength of the OPA output is adjusted, we always keep the probe beam spot size significantly smaller than the pump beam so that the probe beam experiences a nearly uniform change in the refractive index in the transverse dimensions. The angles of incidences are $15^{\circ}$ and $10^{\circ}$ for the pump and probe, respectively. The transmitted probe light is coupled to an optical spectrum analyzer via a multimode fiber with a $50 \mu \mathrm{m}$ core diameter. The commercially available ITO thin film (PGO GmbH) has a thickness of $310 \mathrm{~nm}$ and is deposited on a 1.1-mm-thick glass substrate. We sandwich two such ITO films to make the $620 \mathrm{~nm}$ thick ITO sample by using a customized sample holder with adjustable tightening screws (See Supplementary Note 6). We use a translation stage to control the delay time between the pump and the probe beams. The experimental setup is presented in Supplementary Note 1.

\section{Data availability}

All data supporting this study are available from the corresponding author upon request

\section{Code availability}

All relevant computer codes supporting this study are available from the corresponding author upon request.

Received: 6 March 2020; Accepted: 19 March 2020; Published online: 01 May 2020

\section{References}

1. Akhmanov, S., Sukhorukov, A. \& Chirkin, A. Nonstationary phenomena and space-time analogy in nonlinear optics. Sov. Phys. JETP 28, 748-757 (1969).

2. Kolner, B. H. Space-time duality and the theory of temporal imaging. IEEE $J$. Quant. Electron 30, 1951-1963 (1994).

3. Xiao, Y., Maywar, D. N. \& Agrawal, G. P. Reflection and transmission of electromagnetic waves at a temporal boundary. Opt. Lett. 39, 574-577 (2014).

4. Xiao, Y., Agrawal, G. P. \& Maywar, D. N. Spectral and temporal changes of optical pulses propagating through time-varying linear media. Opt. Lett. 36, 505-507 (2011). 
5. Plansinis, B. W., Donaldson, W. R. \& Agrawal, G. P. What is the temporal analog of reflection and refraction of optical beams? Phys. Rev. Lett. 115, 183901 (2015).

6. Mendonça, J., Guerreiro, A. \& Martins, A. M. Quantum theory of time refraction. Phys. Rev. A 62, 033805 (2000).

7. Mendonça, J. \& Guerreiro, A. Time refraction and the quantum properties of vacuum. Phys. Rev. A 72, 063805 (2005)

8. Boyd, R. W. Nonlinear optics. 3rd edn (Elsevier, 2003).

9. Alam, M. Z., Schulz, S. A., Upham, J., De Leon, I. \& Boyd, R. W. Large optical nonlinearity of nanoantennas coupled to an epsilon-near-zero material. Nat. Photonics 12, 79-83 (2018).

10. Caspani, L. et al. Enhanced nonlinear refractive index in $\varepsilon$-near-zero materials. Phys. Rev. Lett. 116, 233901 (2016).

11. Reshef, O., De Leon, I., Alam, M. Z. \& Boyd, R. W. Nonlinear optical effects in epsilon-near-zero media. Nat. Rev. Mat. 4, 535-551 (2019).

12. Yu, Z. \& Fan, S. Complete optical isolation created by indirect interband photonic transitions. Nat. Photonics 3, 91-94 (2009).

13. Shaltout, A. M., Shalaev, V. M. \& Brongersma, M. L. Spatiotemporal light control with active metasurfaces. Science 364, eaat3100 (2019).

14. Pendry, J. Time reversal and negative refraction. Science 322, 71-73 (2008).

15. Fang, K., Yu, Z. \& Fan, S. Realizing effective magnetic field for photons by controlling the phase of dynamic modulation. Nat. Photonics 6, 782-787 (2012).

16. Lustig, E., Sharabi, Y. \& Segev, M. Topological aspects of photonic time crystals. Optica 5, 1390-1395 (2018).

17. Williamson, I. A. \& Fan, S. Broadband optical switch based on an achromatic photonic gauge potential in dynamically modulated waveguides. Phys. Rev. Appl. 11, 054035 (2019).

18. Wilson, C. M. et al. Observation of the dynamical casimir effect in a superconducting circuit. Nature 479, 376-379 (2011).

19. Yacomotti, A. M. et al. Nonadiabatic dynamics of the electromagnetic field and charge carriers in high-q photonic crystal resonators. Phys. Rev. Lett. 96, 093901 (2006)

20. Preble, S. F., Xu, Q. \& Lipson, M. Changing the colour of light in a silicon resonator. Nat. Photonics 1, 293-296 (2007).

21. Upham, J., Tanaka, Y., Asano, T. \& Noda, S. On-the-fly wavelength conversion of photons by dynamic control of photonic waveguides. Appl. Phys. Express 3, 062001 (2010).

22. Kampfrath, T. et al. Ultrafast adiabatic manipulation of slow light in a photonic crystal. Phys. Rev. A 81, 043837 (2010).

23. Castellanos Muñoz, M., Petrov, A. Y. \& Eich, M. All-optical on-chip dynamic frequency conversion. Appl. Phys. Lett. 101, 141119 (2012).

24. Kondo, K. \& Baba, T. Dynamic wavelength conversion in copropagating slowlight pulses. Phys. Rev. Lett. 112, 223904 (2014).

25. Dekker, R. et al. Ultrafast kerr-induced all-optical wavelength conversion in silicon waveguides using $1.55 \mu \mathrm{m}$ femtosecond pulses. Opt. Express 14, 8336-8346 (2006).

26. Agrawal, G. P., Baldeck, P. \& Alfano, R. Temporal and spectral effects of cross-phase modulation on copropagating ultrashort pulses in optical fibers. Phys. Rev. A 40, 5063-5072 (1989)

27. Li, J., Olsson, B.-E., Karlsson, M. \& Andrekson, P. A. Otdm add-drop multiplexer based on xpm-induced wavelength shifting in highly nonlinear fiber. J. Lightwave Technol. 23, 2654-2661 (2005).

28. Mehta, P., Healy, N., Day, T., Badding, J. V. \& Peacock, A. Ultrafast wavelength conversion via cross-phase modulation in hydrogenated amorphous silicon optical fibers. Opt. Express 20, 26110-26116 (2012).

29. Bloembergen, N. Laser-induced electric breakdown in solids. IEEE J. Quant. Electron. 10, 375-386 (1974).

30. Yablonovitch, E. Self-phase modulation of light in a laser-breakdown plasma. Phys. Rev. Lett. 32, 1101-1104 (1974).

31. Wood, W. M., Siders, C. \& Downer, M. Measurement of femtosecond ionization dynamics of atmospheric density gases by spectral blueshifting. Phys. Rev. Lett. 67, 3523-3526 (1991).

32. Lopes, N. et al. Laser pulse frequency up-shifts by relativistic ionization fronts. Europhys. Lett. 66, 371-377 (2004).

33. Fan, L. et al. Integrated optomechanical single-photon frequency shifter. Nat. Photonics 10, 766-770 (2016).

34. Del'Haye, P. et al. Optical frequency comb generation from a monolithic microresonator. Nature 450, 1214-1217 (2007).

35. Li, Q., Davanço, M. \& Srinivasan, K. Efficient and low-noise single-photonlevel frequency conversion interfaces using silicon nanophotonics. Nat. Photonics 10, 406-414 (2016).

36. Gaafar, M. A. et al. Reflection from a free carrier front via an intraband indirect photonic transition. Nat. Commun. 9, 1447 (2018)

37. Beggs, D. M., Krauss, T. F., Kuipers, L. \& Kampfrath, T. Ultrafast tilting of the dispersion of a photonic crystal and adiabatic spectral compression of light pulses. Phys. Rev. Lett. 108, 033902 (2012).

38. Lee, K. et al. Linear frequency conversion via sudden merging of meta-atoms in time-variant metasurfaces. Nat. Photonics 12, 765-773 (2018).
39. Shcherbakov, M. R. et al. Photon acceleration and tunable broadband harmonics generation in nonlinear time-dependent metasurfaces. Nat. Commun. 10, 1345 (2019).

40. Gaburro, Z. Photonic energy lifters and event horizons with time-dependent dielectric structures. J. Nanophotonics 2, 021853 (2008).

41. Silveirinha, M. \& Engheta, N. Tunneling of electromagnetic energy through subwavelength channels and bends using $\varepsilon$-near-zero materials. Phys. Rev. Lett. 97, 157403 (2006).

42. Engheta, N. Pursuing near-zero response. Science 340, 286-287 (2013).

43. Liberal, I. \& Engheta, N. Near-zero refractive index photonics. Nat. Photonics 11, 149-158 (2017).

44. Alam, M. Z., De Leon, I. \& Boyd, R. W. Large optical nonlinearity of indium tin oxide in its epsilon-near-zero region. Science 352, 795-797 (2016).

45. Kinsey, N. et al. Epsilon-near-zero al-doped zno for ultrafast switching at telecom wavelengths. Optica 2, 616-622 (2015).

46. Ferrera, M. \& Carnemolla, E. G. Ultra-fast transient plasmonics using transparent conductive oxides. J. Optics 20, 024007 (2018).

47. Capretti, A., Wang, Y., Engheta, N. \& Dal Negro, L. Enhanced third-harmonic generation in si-compatible epsilon-near-zero indium tin oxide nanolayers. Opt. Lett. 40, 1500-1503 (2015)

48. Agrawal, G. P. Nonlinear fiber optics. in Nonlinear Science at the Dawn of the 21st Century. Lecture Notes in Physics, Vol. 542 (Springer, 2000).

49. Gui, Y. et al. Towards integrated metatronics: a holistic approach on precise optical and electrical properties of indium tin oxide. Sci. Rep. 9, 1-10 (2019).

50. Yang, Y. et al. Femtosecond optical polarization switching using a cadmium oxide-based perfect absorber. Nat. Photonics 11, 390-395 (2017).

51. Saglamyurek, E. et al. Quantum storage of entangled telecom-wavelength photons in an erbium-doped optical fibre. Nat. Photonics 9, 83-87 (2015).

52. Yanik, M. F. \& Fan, S. Stopping light all optically. Phys. Rev. Lett. 92, 083901 (2004).

53. Yoo, S. B. Wavelength conversion technologies for wdm network applications. J. Lightwave Technol. 14, 955-966 (1996).

54. Caloz, C. et al. Electromagnetic nonreciprocity. Phys. Rev. Appl. 10, 047001 (2018).

55. Sounas, D. L., Caloz, C. \& Alu, A. Giant non-reciprocity at the subwavelength scale using angular momentum-biased metamaterials. Nat. Commun. 4, 2407 (2013).

56. Giergiel, K., Dauphin, A., Lewenstein, M., Zakrzewski, J. \& Sacha, K. Topological time crystals. N. J. Phys. 21, 052003 (2019).

57. Bruno, V. et al. Broad frequency shift of parametric processes in epsilon-nearzero time-varying media. Appl. Sci. 10, 1318 (2020).

\section{Acknowledgements}

R.W.B. C.L., Y.Z. and A.E.W. acknowledge support from DARPA (grant No. W911NF18-0369). O.R. acknowledges the support of the Banting Postdoctoral Fellowship from the Natural Science and Engineering Research Council, Canada. This work was supported by the US Office of Naval Research and the Natural Science and Engineering Research Council, Canada. Note: while our paper was in review, a complementary paper was submitted and published ${ }^{57}$. In contrast to our work, they demonstrate wavelength conversion using four-wave mixing process in a time-varying ENZ thin film.

\section{Author contributions}

M.Z.A., J.U., and R.W.B. conceived the work after serendipitous observation of the effect in the laboratory by M.Z.A. M.Z.A. performed the first set of laboratory tests. M.Z.A., Y.Z., O.R., and J.U. designed the experiment. Y.Z. with help from M.Z.A., M.K., O.R., J.U., and C.L. performed laboratory measurements. Y.Z. with help from M.Z.A. performed data analysis and developed the numerical model. Y.Z and M.Z.A. wrote the first draft. All authors contributed to the discussion of the results and the preparation of the final version of the manuscript. A.E.W. and R.W.B. supervised the work.

\section{Competing interests}

The authors declare no competing interests.

\section{Additional information}

Supplementary information is available for this paper at https://doi.org/10.1038/s41467 020-15682-2.

Correspondence and requests for materials should be addressed to Y.Z.

Peer review information Nature Communications thanks the anonymous reviewers for their contribution to the peer review of this work. Peer reviewer reports are available.

Reprints and permission information is available at http://www.nature.com/reprints

Publisher's note Springer Nature remains neutral with regard to jurisdictional claims in published maps and institutional affiliations. 
(c) (i) Open Access This article is licensed under a Creative Commons Attribution 4.0 International License, which permits use, sharing, adaptation, distribution and reproduction in any medium or format, as long as you give appropriate credit to the original author(s) and the source, provide a link to the Creative Commons license, and indicate if changes were made. The images or other third party material in this article are included in the article's Creative Commons license, unless indicated otherwise in a credit line to the material. If material is not included in the article's Creative Commons license and your intended use is not permitted by statutory regulation or exceeds the permitted use, you will need to obtain permission directly from the copyright holder. To view a copy of this license, visit http://creativecommons.org/ licenses/by/4.0/.

(C) The Author(s) 2020 